\title{
COVID-19 AND HEMATOPOIETIC STEM CELL TRANSPLANTATION IN BRAZIL: ARE WE COPING?
}

\author{
EDITORIAL
}

Antonio Vaz de Macedo $0^{1,2,3}$

1- Hematology Clinic, Hospital da Polícia Militar, Belo Horizonte, Minas Gerais, Brazil. 2- Bone Marrow Transplant Unit, Hospital Luxemburgo, Instituto Mário Penna, Belo Horizonte, Minas Gerais, Brazil. 3- Principles and Practice of Clinical Research Program, ECPE, Harvard T.H. Chan School of Public Health, Boston, Massachusetts, USA.

Correpondence to: antoniovmac@hotmail.com

Ever since the first coronavirus disease 2019 (COVID-19) case was reported in Brazil in late February, 2020, the pandemic has reached virtually every corner of the country. Spanning continental dimensions, it is by far the most affected country in Latin America, with the second highest death toll in the world (almost 150,000), and almost 5 million confirmed cases as of October 4, second only to the Unites States (https://coronavirus.jhu.edu/map. html). The actual death toll might actually be higher, due to both limited testing and inconsistency in nationwide cause-of-death reports. Apart from the available evidence on the benefit of dexamethasone (and other steroids) in patients under respiratory support, no effective treatment has thus far been shown to reduce mortality in this disease.[1] Likewise, despite global efforts and billion dollar investments, none of the dozens of vaccines currently being tested in humans are expected to be available at scalable and nationwide levels within the next few months.

To date, the vast majority of the data gathered about the epidemiology, clinical course, prevention, and treatment of COVID-19 have come from studies of non-transplant and non-immunocompromised patients. Little is known about the disease in the hematopoietic stem cell transplantation (HSCT) setting, though one might presume HSCT patients to be at an increased risk of serious complications and death due to COVID-19. As a result of a myriad of collaborative efforts, expert panels have been issuing several recommendations in this regard (https://www.sbtmo.org.br/saibamais/covid-19-e-tmo). As a rule, in general terms, clinicians should follow the available guidelines for managing COVID-19 in non-transplant patients, with stricter attention, though, to the selection of transplant candidates and donors, as well as to the molecular and serologic monitoring of severe acute respiratory syndrome coronavirus 2 (SARS-Cov2) infection among patients, donors, caregivers, and healthcare professionals. Treating COVID-19 in the HSCT scenario may be particularly challenging, given the common coexistence of comorbidities, transplant-related cytopenias, potential drug-to-drug interactions, overlapping toxicities, and the need for chronic immunosuppressive therapy to prevent graft rejection and graft-versus-host disease in the allogeneic setting. This complex interaction between both the baseline disease and the various aspects of the procedure itself makes any assessment of the actual attributable impact of HSCT on the severity of COVID-19 even more demanding (https://www.covid19treatmentguidelines.nih.gov/ whats-new/). Specific guidelines, including those of Machado C., 2020, on behalf of the Brazilian Society of Bone Marrow Transplantation (SBTMO), coupled with several freely-available HSCT and COVID-19-related webinars, online resources, and continuing education programs, have provided practical, evidence-based guidance to caregivers and healthcare professionals[2] . One has to bear in mind, though, the rapid and ever-changing landscape of the pandemic. This has posed an increasing demand on the transplant community, in its effort to keep itself updated with the latest COVID-19 releases.

While grappling with the best response toward the spread of SARS-Cov2 across HSCT centers in the past six months, many have found themselves adapting their transplant routines and procedures. In a recent nationwide survey (unpublished data) led by Duarte, FB., 2020, on behalf of the SBTMO, from May to June, with the aim of evaluating the overall impact of the SARS-CoV-2 pandemic on Brazilian HSCT center routines and protocols until then, a $60 \%$ response rate to the questionnaires (out of a total of 86 certified centers) was obtained, comprising approximately $85 \%$ of the adult and pediatric transplant activity in the country. In this study, the authors noted a decrease between $50 \%$ and $75 \%$ in the general HSCT activity in $59.2 \%$ of all participating centers. All such centers fol- 
lowed some kind of evidence-based guidance, mainly that from the SBTMO ( $>90 \%$ of cases). A minority of them $(5-12 \%)$ completely discontinued their transplant programs, $30-43 \%$ cut by half their transplant rates, whereas $6-12 \%$ did not perceive any substantial changes in this respect. The authors highlighted the initial lack of universal SARS-Cov2 testing of donors and of asymptomatic patients prior to transplant, the main reasons for which seemed to be the lack of access to or delay in obtaining the results of real-time polymerase chain reaction (RT-PCR) tests, and/or of an adequate facility for the collection of samples. This also hampered testing among the healthcare professionals in charge of such patients. Even so, infection rates among these were reported by up to $73 \%$ of centers in June. COVID-19 diagnosis raised from one month to the other, with up to $23 \%$ and $48 \%$ of the analyzed centers reporting cases during hospital stay and post-discharge periods, respectively. Death reports due to COVID-19 raised from $13 \%$ to $18 \%$ of centers from May to June. As in other non-transplant scenarios, fever and cough were among the most prominent symptoms found, with no distinctive features noted. Azithromycin was the most commonly used treatment (75\% of centers), and immunosuppressants, when used, were kept unaltered in most cases. This survey helped depict some of the major barriers to the optimal management and preventive measures noted in the first few months of the pandemic in the country. One might expect that, given the global learning curve acquired since the beginning of the pandemic, with more widespread testing, use of personal protective equipment, and refinement of institutional policies directed toward SARS-Cov2 infection, some of such barriers may have been (or are bound to be) overcome from July onwards, with better results in the months to come.

Further data from the Brazilian Transplant Registry (RBT) of the Brazilian Association of Organ Transplantation (ABTO) from the first semester (http://abto.org. $\mathrm{br} /$ ) indicate a $20 \%$ reduction in the HSCT rate, year on year, which corresponds to an absolute reduction from 1621 to 1302 in the number of transplants, with an even greater drop in the autologous setting (26\% compared to a $10 \%$ decrease in the allogeneic subgroup). A possible explanation for this might be the chronic and somewhat less urgent nature of the main indication for autologous transplant in our country, multiple myeloma. One might, perhaps, have expected a greater drop in transplant numbers due to the pandemic. Caution is needed, though, in the interpretation of these findings, given the underreporting (of over $10 \%$ compared to last year) by the participating sites (HSCT rates might actually have been higher in the first half of 2020). Data on the third quarter of the year are expected for the middle of October.

In a year in which Brazil's Unified Health System (Sistema Único de Saúde- SUS), recognized by the World Health Organization (WHO) as the world's largest publicly funded universal healthcare system to date, upon which depend virtually $75 \%$ of the country's population, is supposed to be celebrating its 30th Birthday (http://www.planalto.gov.br/ccivil_03/leis/ I8080.htm), both the public and private healthcare sectors (including individual health plans) are faced with the utmost challenge of providing the necessary support to the country's more than 210 million citizens. To make matters worse, health policy has somewhat turned into health politics, which surely undermines much of the concerted efforts needed to counteract the COVID-19 pandemic. Another challenge is that of the need for circumventing the poor housing conditions of many HSCT patients and of their family members within the context of the public healthcare system. This greatly reflects the worrisome socioeconomic and educational status of the most underserved racial and ethnic minorities in the country. Social support systems that are able to provide a solid safety net for a close post-HSCT follow-up are, therefore, a must.

Nonetheless, there seems to be light at the end of the tunnel. Ironically, the COVID-19 pandemic has torn down borders and brought the transplant community even closer together. While social distancing and virtual encounters have become "the rule", in order to keep pace with the ever-changing COVID-19 landscape, we have often found ourselves sharing the intimacy of our homes, turning remote interaction and collaboration into seemingly face-to-face ones. Stronger ties have developed, fostering a myriad of nationwide collaborative efforts, from basic to clinical research, the results of which are eagerly awaited.

\section{REFERENCES}

1- RECOVERY Collaborative Group, Horby P, Lim WS, Emberson JR, Mafham M, Bell JL, Linsell L, et al. Dexamethasone in Hospitalized Patients with Covid-19 - Preliminary Report. N Engl J Med. 2020 Jul 17:NEJMoa2021436. doi: 10.1056/NEJMoa2021436. Epub ahead of print.

2- Machado, CM. COVID-19 and Hematopoietic Stem Cell Transplantation: Recommendations from the Brazilian Society of Bone Marrow Transplantation (SBTMO). Journal of Bone Marrow Transplantation and Cellular Therapy. 2020, p.1: 67-72. doi: 10.46765/2675-374X.2020V1N1P67-72. 